TRANSACTIONS OF THE

AMERICAN MATHEMATICAL SOCIETY

Volume 187, Is sue 1, 1974

\title{
ON A COMPACTNESS PROPERTY OF TOPOLOGICAL GROUPS
}

\author{
BY
}

S. P. WANG(1)

ABSTRACT. A density theorem of semisimple analytic groups acting on locally compact groups is presented.

Let $G$ and $H$ be locally compact groups with $G$ acting continuously on $H$ as a group of automorphisms. An element $b$ of $H$ is said to be $G$-bounded if the orbit $G b=\{g(b): g \in G\}$ has compact closure in $H$. We write $F_{G}(H)$ for the set of all $G$-bounded elements in $H$. It is very easy to verify that $F_{G}(H)$ is a $G$-invariant subgroup of $H$. However in general, $F_{G}(H)$ is not closed in $H$. In this paper, we shall study the group $F_{G}(H)$ for certain topological groups $G$. Our main result is the following

Theorem. Let $G$ be a semisimple analytic group without compact factors acting on a locally compact group $H$ continuously as a group of automorphisms. If the set $F_{G}(H)$ is dense in $H$, then $G$ acts trivially on $H$.

The theorem generalizes some results in [2], [4] and is closely related to the density property of certain subgroups in semisimple analytic groups without compact factors. The result of Corollaries 4.1 and 4.2 is contained in [2], [4].

In the sequel, we shall use the term " $G$ acts on $H$ " for " $G$ acts on $H$ as a group of automorphisms".

1. Minimally almost periodic groups. Let $G$ be a locally compact group. We recall that $G$ is minimally almost periodic if there are no nontrivial continuous homomorphisms $f: G \rightarrow G^{\prime}$ of locally compact groups such that the closure $\mathrm{Cl}(f(G))$ of $f(G)$ in $G^{\prime}$ is compact. Minimally almost periodic groups have been widely studied. Yet for our need, we shall establish some lemmas concerning minimally almost periodic groups.

Lemma 1.1. Let $G$ be a minimally almost periodic group acting continuously on a locally compact group $H$, and $N$ be a closed G-invariant normal subgroup of $H$.

Received by the editors December 4, 1970 and, in revised form, February 1, 1972.

AMS (MOS) subject classifications (1970). Primary 22D05; Secondary 22E15.

Key words and phrases. $G$-bounded elements, locally compact groups, analytic group, linear Lie groups, almost periodic groups, cross homomorphisms.

(1) Partially supported by NSF Grant GP-667350-1395. The author would like to thank the referee for helpful suggestions. 
If the set $F_{G}(H)$ is dense in $H$, and $G$ acts trivially on both $N$ and $H / N$, then $G$ acts trivially on $H$.

Proof. Let $b$ be any fixed element of $F_{G}(H)$. As $G$ acts trivially on $H / N$, there is a continuous function $f: G \rightarrow N$ such that $g(b)=b f(g), g \in G$. Since $G$ acts trivially on $N$, we have

$$
\begin{aligned}
b f\left(g^{\prime} g\right) & =\left(g^{\prime} g\right) b=g^{\prime}(g b)=g^{\prime}(b f(g)) \\
& =g^{\prime}(b) f(g)=b f\left(g^{\prime}\right) f(g) \quad\left(g^{\prime}, g \in G\right) .
\end{aligned}
$$

Hence $f$ is a continuous homomorphism. We know that $\mathrm{Cl}(G b)$ is compact. $\mathrm{Cl}\left(f(G)\right.$ ), being contained in $b^{-1} \mathrm{Cl}(G b)$, is evidently compact. Therefore $f$ has to be trivial; equivalently $g(b)=b$ for every $g$ in $G$. Since the set $F_{G}(H)$ is dense in $H$, it follows readily that $G$ acts trivially on $H$.

Lemma 1.2. Let $G$ be a connected minimally almost periodic group acting continuously on a locally compact abelian group $A$. If the set $F_{G}(A)$ is dense in $A$, then $G$ acts trivially on $A$.

Proof. First we assume that $A$ is compactly generated. In this case, $A$ has a unique maximal compact subgroup $K$. Obviously $K$ is characteristic, hence $G$ invariant. By a well-known theorem of Iwasawa [3], the automorphism group Aut $(K)$ of $K$ with compact-open topology is totally disconnected, hence $G$ acts trivially on $K$. By Lemma 1.1, we may assume that $K=\{e\}$ and $A$ is an abelian Lie group. Let $A^{\circ}$ be the identity component of $A$. Since $G$ is connected, $G$ acts trivially on $A / A^{\circ}$. Again by Lemma 1.1 , we may even assume that $A$ is connected. Under these additional assumptions, $A=R^{l}$ for some positive integer $l$. Now we pick out a basis $\left\{e_{i}, \cdots, e_{l}\right\}$ of $R^{l}$ from $F_{G}\left(R^{l}\right)$. This is possible because $F_{G}\left(R^{l}\right)$ is dense in $R^{l}$. With respect to this basis, for every $g$ in $G$, we write

$$
g\left(e_{i}\right)=\sum_{j=1}^{l} g_{j i} e_{j} \quad(1 \leq i \leq l),
$$

with $g_{j i}$ in $R$. It is easy to show that the map $g \rightarrow\left(g_{i j}\right)(g \in G)$ is a continuous homomorphism $f$ of $G$ into $G L(l, R)$. Since all the entries $g_{i j}(1 \leq i, j \leq l, g \in G)$ are bounded, we conclude $f(G)$ has compact closure in $\operatorname{GL}(l, R)$. Hence $f$ has to be trivial, and the lemma is proved in case that $A$ is compactly generated. For the general case, $G$ acts trivially on $A / A^{\circ}$, hence $G$ leaves any open subgroup of $A$ invariant. Let $N$ be a compactly generated open subgroup of $A$. Clearly $F_{G}(N)=$ $F_{G}(A) \cap N$ is still dense in $N$. By what we have just proved, $G$ acts trivially on $N$ and by Lemma 1.1, the proposition follows.

Remark. In the preceding lemma, we assume only that the set $F_{G}(A)$ is dense in $A$. In general we do not know whether the set $F_{G}\left(A^{\circ}\right)=F_{G}(A) \cap A^{\circ}$ is dense in $A^{\circ}$. That is why we consider first compactly generated open subgroups of $A$ rather than the subgroup $A^{\circ}$. 
Corollary 1.3. Let $G$ be a connected minimally almost periodic group and $L$ a closed subgroup of $G$ with compact quotient $G / L$. Let $A$ be a locally compact abelian group such that $G$ acts continuously on $A$. If $L$ leaves an element $x$ of $A$ fixed, then $x$ is fixed by $G$.

Proof. Consider the group $\mathrm{Cl}\left(F_{G}(A)\right)$. By Lemma 1.2, $G$ acts trivially on $\mathrm{Cl}\left(F_{G}(A)\right)$. Clearly $x$ lies in $F_{G}(A)$ and the corollary follows.

Corollary 1.3 reveals at least some density property of those subgroups $L$ of $G$ with compact quotient $G / L$. In general, the structure of minimally almost periodic groups is not entirely clear. However for connected groups, we have the following criterion. The result must be known but we offer a proof here for completeness.

Lemma 1.4. Let $G$ be a connected locally compact group. The following statements are equivalent:

(i) $G$ is minimally almost periodic.

(ii) $G$ is an analytic group such that $[G, G]$ is dense in $G$ and $G / R(G)$ bas no compact factors where $R(G)$ is the radical of $G$.

Proof. (i) $\Rightarrow$ (ii) Since $G$ is a connected locally compact group, locally $G$ is the direct product of a compact group and a local Lie group. However $G$ is minimally almost periodic, hence $G$ is a Lie group. Consider then the groups $G / \mathrm{Cl}([G, G])$ and $G / R(G) . G / R(G)$ (resp. $G / C l([G, G])$ is minimally almost periodic semisimple (resp. abelian minimally almost periodic). (ii) follows immediately.

(ii) $\Rightarrow$ (i) By a well-known theorem of von Neumann, any topological group $G$ contains a unique minimal closed normal subgroup $N$ such that $G / N$ is maximally almost periodic, i.e., there is a continuous injection of $G / N$ into a compact group $K$. Hence it suffices to show that $G / N$ is trivial in our case. Clearly $G / N$ still satisfies all the assumptions in (ii). But, by a theorem of Freudenthal, a connected maximally almost periodic locally compact group is the direct product of a compact group and a vector group. Hence one concludes readily that $G / N$ is trivial, i.e., $G=N$ is minimally almost periodic.

2. Cross homomorphisms. Let $G$ be a locally compact group acting on a locally compact abelian group $A$ continuously. A continuous map $f: G \rightarrow A$ is called a cross homomorphism if $f$ satisfies the condition

$$
f\left(g g^{\prime}\right)=g\left(f\left(g^{\prime}\right)\right)+f(g)
$$

for all $g, g^{\prime}$ in $G$. Given any $v$ in $A$, the map $d_{v}: G \rightarrow A$, defined by $d_{v}(g)=g v$ $-v(g \in G)$ clearly is a cross homomorphism. A cross homomorphism $f$ is said to be bomologous to 0 if $f=d_{v}$ for some $v$ in $A$.

Lenma 2.1. Let $G$ be a semisimple analytic group acting on a locally compact abelian group $A$ continuously. Then any cross bomomorphism $f: G \rightarrow A$ is bomologous to 0 . 
Proof. Let $e$ be the identity element of $G$. Since $f$ is a cross homomorphism, $f(e)=0$. Hence $f(G)$ is contained in $A^{\circ}$ because $f$ is continuous. Therefore we may even assume that $A$ is connected. Let $K$ be the unique maximal compact subgroup of $A$. Clearly $K$ is $G$-invariant and $G$ acts trivially on $K . f$ induces then a cross homomorphism $\bar{f}: G \rightarrow A / K . A / K$ is isomorphic to $R^{l}$ for some positive integer $l$. It is well known that $\bar{f}$ is homologous to 0 . Hence there exists an element $v$ in $A$ such that

$$
f(g) \equiv g v-v \quad(\bmod K), \quad g \in G .
$$

Let $f_{1}: G \rightarrow K$ be the map defined by

$$
f_{1}(g)=f(g)-g v+v, \quad g \in G .
$$

One verifies readily that $f_{1}$ is a cross homomorphism. Since $G$ acts trivially on $K$, $f_{1}$ is a homomorphism, hence $f_{1}(G)=\{0\}$. Thus $f=d_{v}$ is homologous to 0 .

3. Linear Lie groups. Let $\mathrm{GL}(n, C)$ (resp. $g l(n, C)$ ) be the group of all $n$ by $n$ nonsingular complex matrices (resp. the Lie algebra of all $n$ by $n$ complex matrices). Clearly $g \mathrm{~L}(n, C)$ is the Lie algebra of $\mathrm{GL}(n, C)$ and the exponential map exp: $g \mathfrak{L}(n, C) \rightarrow \mathrm{GL}(n, C)$ is just the usual one. Let $\lambda$ be any positive number. We denote by $g l(n, C ; \lambda)$ the set of all elements $X$ in $g l(n, C)$ such that the imaginary parts of all the eigenvalues of $X$ lie in the open interval $(-\lambda, \lambda)$. Let $G$ be any $L_{i e}$ subgroup of $G L(n, C)$ and $g$ its Lie algebra. We write $g_{\lambda}, G_{\lambda}$ and $\exp _{\lambda}$ for $g \cap g L(n, C ; \lambda), \exp \left(g_{\lambda}\right)$ and the restriction of $\exp _{\lambda}$ on $g_{\lambda}$ respectively.

Lemma 3.1 [4]. The maps $\exp _{\lambda}(0<\lambda \leq \pi)$ are diffeomorphisms.

Proposition 3.2. Let $G$ be a semisimple analytic subgroup of $\mathrm{GL}(n, C)$ and $H$ a Lie subgroup of $\mathrm{GL}(n, C)$. Suppose that

(i) $G$ bas no compact factors,

(ii) $G$ normalizes $H$, and

(iii) $F_{G}(H)$ is dense in $H$ where $G$ acts on $H$ through conjugation.

Then $G$ centralizes $H$.

Proof. Let $\lambda$ be any positive number smaller than $\pi$. By Lemma 3.1, $\exp _{\lambda}: \mathscr{G} \rightarrow H_{\lambda}$ is a diffeomorphism. Clearly $G_{\lambda}$ is $G$-invariant under conjugation. Since $F_{G}(H)$ is dense in $H$, there is a basis $\left\{X_{1}, \cdots, X_{r}\right\}$ of $\mathscr{G}$ such that $X_{i} \in \mathscr{G}_{\lambda}$ and $\exp X_{i} \epsilon$ $F_{G}(H)(1 \leq i \leq r)$. Let $A d$ be the adjoint representation of $G L(n, C)$ on $g^{l}(n, C)$. Then with respect to this basis, all elements in the group $\left.\operatorname{Ad}(G)\right|_{G}$ have bounded entries because $\exp X_{i} \in F_{G}(H)(1 \leq i \leq n)$ and $\exp _{\lambda}$ is a diffeomorphism. Hence $\left.\operatorname{Ad}(G)\right|_{\mathfrak{h}}$ has compact closure. By (i) and Lemma $1.4, G$ centralizes $H^{\circ}$. Clearly $G$ acts trivially on $H / H^{\circ}$ for $H / H^{\circ}$ is discrete and $G$ is connected. By Lemma 1.1, $G$ acts trivially on $H$, therefore $G$ centralizes $H$. 
4. Proof of the theorem. We prove the theorem in several steps.

(i) $G$ leaves invariant any open subgroup of $H$. Since $H / H^{\circ}$ is discrete and $G$ is connected, $G$ acts trivially on $H / H^{\circ}$. Clearly $H^{\circ}$ is contained in any open subgroup of $H$. Hence (i) follows easily.

(ii) We may assume that $H$ is an analytic group. Let $H_{1}$ be an open subgroup of $H$ such that $H_{1}$ is a projective limit of Lie groups. Let $K$ be a normal compact subgroup of $H_{1}$ such that $H_{1} / K$ is a Lie group. Then consider $H_{2}=H_{1}^{\circ} K . H_{2}$ is again an open subgroup of $H$. It is well known that a connected locally compact group has a unique maximal compact normal subgroup. It follows that $\mathrm{H}_{2}$ also has a unique maximal normal compact subgroup $L$. By (i) $H_{2}$ is $G$-invariant, hence $L$ is also $G$-invariant. Since $L$ is compact, Aut $(L)^{\circ}=$ the inner automorphism group by a theorem of Iwasawa [3]. Therefore Aut $(L)^{\circ}$ is compact. The action of $G$ on $L$ is induced by a continuous homomorphism $f: G \rightarrow$ Aut $(L)$. Clearly $f(G)$, being contained in Aut $(L)^{\circ}$, has compact closure. By Lemma $1.4, f(G)$ is trivial, i.e., $G$ acts trivially on $L$. Therefore by Lemma 1.1 , we may even assume that $H=H_{2} / L$ is an analytic group.

(iii) By (ii) we assume further that $H$ is an analytic group. Let $M=G \cdot H$ be the semidirect product of $G$ and $H$. Let $A d$ be the adjoint representation of $M$ on its Lie algebra. Passing over to $\operatorname{Ad}(M)$, by Proposition 3.2, one concludes that given any $b \in H$

$$
g(b)=b s(g), \quad g \in G,
$$

where $s(g)$ is in the center $Z(H)$ of $H$. By a direct calculation, $s: G \rightarrow Z(H)$ is a cross homomorphism. By Lemma 2.1, $s$ is homologous to 0 . Hence there is $z \in Z(H)$ with $s(g)=g\left(z^{-1}\right) z$ for all $g \in G$. Now consider the element $b z$. Clearly $g(b z)=b z$ for all $g \in G$. Let $F$ be the set of all fixed points of $H$. Clearly $F$ is a closed subgroup of $H$. By what we have just proved, $F \cdot Z(H)=H$. Hence $F$ is normal and $H / F$ is abelian. By Proposition 1.2, $G$ acts trivially on $H / F$. By Lemma 1.1, $G$ acts trivially on $H$. Therefore the proof of the theorem is hereby completed.

Corollary 4.1. Let $G$ be analytic semisimple group without compact factors, and $g$ an element of $G$. If the conjugacy class $\left\{x g x^{-1}: x \in G\right\}$ bas compact closure in $G, g$ is in the center $Z(G)$ of $G$.

Proof. $G$ acts on $G$ through conjugation. By the theorem $F_{G}(G)=Z(G)$. Clearly $g$ is in $F_{G}(G)$.

Corollary 4.2. Let $G$ be an analytic semisimple group without compact factors and $\alpha$ an automorpbism of $G$. If the subset $\left\{a(g) g^{-1}: g \in G\right\}$ bas compact closure then $a$ is the identity map. 
Proof. Let $\omega: G \rightarrow \operatorname{Aut}(G)$ be the homomorphism defined by $\omega(g)(x)=$ $g \times g^{-1},(g, x \in G)$. Clearly $G$ acts on Aut $(G)$ through $\omega$ and conjugation, and $\alpha \in F_{G}($ Aut $(G))$. By the theorem, $G$ leaves $a$ fixed, i.e. $\omega(\alpha(g))=a \omega(g) a^{-1}=$ $\omega(g)$ for all $g$ in $G$. It follows then $\alpha(g) g^{-1}$ is in the center $Z(G)$ of $G$ and the map $g \rightarrow a(g) g^{-1}(g \in G)$ is a homomorphism of $G$ into $Z(G)$. Since $G$ is semisimple, this map has to be trivial. Therefore $a(g)=g$ for all $g$ in $G$, i.e., $\alpha$ is the identity map of $G$.

\section{REFERENCES}

1. R. Baer, Finiteness properties of groups, Duke Math. J. 15 (1948), 1021-1032. MR 10, 352.

2. M. Goto, $A$ remark on a theorem of $A$. Weil, Proc. Amer. Math. Soc. 20 (1969), 163-165. MR 38 \#1211.

3. K. Iwasawa, On some types of topological groups, Ann. of Math. (2) 50 (1949), 507-558. MR 10, 679.

4. J. Tits, Automorphismes à déplacement borné des groupes de Lie, Topology 3 (1964), 1-11. MR $28 \# 2170$.

5. S. P. Wang, Compactness properties of topological groups, Trans. Amer. Math. Soc. 154 (1971), 301-314. MR $42 \# 6152$.

DEPARTMENT OF MATHEMATICS, PURDUE UNIVERSITY, LAFAYETTE, INDIANA 47907 